\title{
Strengthening surgical handover: Developing and evaluating the effectiveness of a handover tool to improve patient safety.
}

\author{
Nizar Din, Shahrzad Ghaderi, Rachel O'Connell, Tayo Johnson - Ashford and St Peter's NHS Foundation Trust
}

\begin{abstract}
The European Working Time Directive and economic challenges have led to a paradigm of shift-work becoming common, and yet the continuation of high-quality patient care remains paramount. Effective and safe transfer of clinical information is critical as emphasised by the Royal College of Surgeons document 'Safe handover: Guidance from the Working Time Directive working party (March 2007)'. The aim of this project was to design and implement a handover proforma in order to deliver a more efficient and safer system for patient care over the weekend. The surgical weekend handover proforma was designed following consultation with nursing and medical colleagues. It included a traffic light scoring system to alert the on-call team of the urgency of clinical review. An educational session was delivered to junior doctors on the surgical rotation to ensure accurate completion of the proforma. All trainee surgical doctors from Foundation Year 1 to Specialist Registrars were asked to complete a pre- and post-intervention questionnaire. Improvement was noted in all categories measured. $85 \%$ of the firms were using the new surgical weekend handover sheet. $78 \%$ of junior doctors were confident in understanding the patient's condition and executing the clinical jobs faster, with ward rounds lasting less than 3 hours. On average, $20 \%$ of patients were discharged per weekend during the trial period. Robust patient handover is vital to maintain patient safety and avoid adverse events. Our findings support the use of a surgical proforma to provide a consistent and structured approach to inpatient handover during the weekend.
\end{abstract}

\section{Problem}

St Peter's hospital (SPH), Chertsey, England is a 400-bed district general hospital providing a range of acute care services including Accident and Emergency. Whilst there is a robust handover of acute surgical admissions twice a day, no system exists for inpatients at weekends, some of which have complex surgical and medical pathologies. Furthermore, at weekends, there is a reduced workforce to review general surgical patients and to manage new acute general surgical admissions. The clinical care for inpatients may subsequently be compromised by lack of knowledge of the patient, their reason for admission and their comorbidities (1).

The aim of this quality improvement project was to create a robust handover system for general surgical patients over the weekend. We evaluated handover before and after the introduction of a paper handover tool created to improve patient safety.

\section{Background}

The European Working Time Directive and economic challenges have led to a paradigm of shift-work becoming common, and yet the continuation of high-quality patient care remains paramount. Many incidents in the clinical environment have been attributed to communication breakdown. Effective and safe transfer of clinical information is critical as emphasised by the Royal College of Surgeons (2). Clinical handover provides a platform to facilitate the continuity of patient information transfer and helps identify and anticipate potential problems for the forthcoming shift. However, handovers are largely informal and the quality is variable and at worst can compromise patient care.

\section{Baseline}

This project was performed at St Peter's hospital and involved upper gastrointestinal, colorectal, breast and vascular surgery. Registrars, senior house officers, house officers (FY1-FY2) and nursing staff were involved.

Pre-intervention data was compiled assessing the proportion of patients with a documented weekend handover. An online questionnaire was sent to all current surgical trainees of all levels of experience (from FY1 to registrars) to gauge their confidence in performing weekend responsibilities that involved managing patients on the ward, their understanding of patient clinical information during the weekend and their estimated duration of weekend ward-rounds. A $65 \%$ response rate was achieved. Opinions from nursing staff were sought to gain comprehensive insight. Quantitative data detailing the proportion of weekend patients being discharged was obtained.

Results showed that only $35 \%$ of patients had a weekend handover sheet. The more junior surgical trainees (FY1 to core surgical trainees) lacked confidence in executing clinical care and took a long time in executing clinical tasks and occasionally did not understand the clinical situation of the patient. Weekend wardrounds took more than 3.5 hours on average with less than $5 \%$ of surgical patients being discharged over the weekend. Almost 100\% of respondents agreed that a proforma with specifically defined categories should be introduced to improve the weekend wardround.

\section{Design}


Following analysis of the questionnaires, consultation with senior colleagues and a nursing and doctor forum, a weekend handover proforma was created (figure1).

The handover proforma includes a 'traffic light' code system to visually highlight the clinical urgency for the patient to be reviewed over the weekend:

o RED: Patient must be reviewed, patient at risk

o YELLOW: Patient stable but not ready for discharge over the weekend

o GREEN: Patient is medically fit, social/other issues are prohibiting discharge.

o BLUE: Patient can be discharged over the weekend.

All blue-coded patients must have discharge summaries completed by the patient's host team, therefore reducing the burden on the oncall team and providing an accurate summary of the inpatient stay.

The aim of the text spaces for admission summary, patient history and potential concerns, investigations and radiology were to provide the assessing doctor with information about the patient 'at a glance'. The text box entitled 'jobs' allowed the patient's usual team to communicate the clinical tasks needed to be completed for the patient over the weekend.

\section{Strategy}

An educational session aided introduction of the handover and aimed to encourage the junior doctors to complete the handover proforma for each patient on a Friday, ready for the weekend. Verbal, email, and poster communication as well as consultant support ensured appropriate advertising of the proforma. Following the pre-intervention questionnaire a pilot trial was performed over 4 weekends. A post-intervention questionnaire was sent out to surgical doctors. The number of weekend patients being discharged over the weekend before and after the intervention was obtained. Results of the intervention were emailed to surgical doctors and suggestions for additional improvements were welcomed.

\section{Post-Measurement}

The results demonstrated that $85 \%$ of the surgical patients had a surgical proforma for the weekend on-call team post-intervention. $70 \%$ of respondents $(n=21)$ were confident in understanding the patient's clinical condition and executed the clinical jobs faster (Figures 2,3). Weekend ward-rounds lasted less than 3 hours on average with $20 \%$ of patients successfully discharged every weekend, post-intervention. With these new changes, the principle problem relates to compliance and the accurate completion of the new handover proforma. A summary of the results pre- and postquality improvement intervention are displayed in figure 4.

See supplementary file: BMJ Safety-diagram results.docx

\section{Lessons and Limitations}

The importance of accurate weekend handover is highlighted in a study by Freemantle et al (3) that looked into 14 million admissions to English NHS hospitals in the financial year of 2009/2010. 187,337 deaths occurred within 30 days of admission in England. When the authors looked at factors associated with risk they found that a patient admitted on a Sunday had $16 \%$ increased risk of dying following admission compared to a person admitted on a Wednesday. Whilst confounding factors exist, a significant cause is attributable to communication and handover. Handover is a fundamental part of modern surgery. We now work in shift patterns and precise handover is vital in protecting patient safety and improving clinical outcomes.

Junior doctors are in a unique position to experience first hand and assess the strengths and weaknesses of existing systems of management and can help provide a fresh outlook on existing problems. With resource limitations, leadership in a doctor's early career is becoming increasingly important to help change practice and is listed as part of good practice by the General Medical Council (4). As this project demonstrates, large and significant changes in practice led by junior doctors are possible without significant input from management or consultants. However, a successful initiative requires adequate education, repeated advertisement and at least some senior-level support for its implementation.

One limitation of this project is that the study commenced at the beginning of the training cycle for all junior surgical doctors and may have led to a potential bias of the results. Firstly because the majority of the junior doctors may have had reduced confidence levels even prior to the intervention, as many of them would have begun their first job post-graduation and hence unfamiliarity and compliance with completion of the forms may have been poor, contributing to possible skewed results. Consequently, this intervention will be revisited at the end of the rotational year. In the future, email reminders about the correct completion of the proforma will help maintain quality and compliance rates. Another learning point is that quality improvement projects are a dynamic process. Initially, the handover proforma was detailed and time consuming to fill in. We adjusted this following continuous feedback from trainees to make it simple and not too laborious. Following numerous modifications, the proforma sheet became a simple and easy form to fill in, whilst being detailed enough to serve its purpose. Another point to highlight is the average percentage of patients being discharged over the weekend (Figure 4). The results showed that during the four-week trial period, there was a marked increase in successful discharge but potential confounding factors such as higher proportion of day case patients may have contributed to these figures. It should be noted that a small sample was analysed and the period of the studies was short. Consequentially, it would be interesting to analyse the change over a longer period of time.

\section{Conclusion}

Our findings support the use of a surgical proforma by providing a consistent and structured approach to handover, coupled with an educational session. This allows transfer of information in a time- 
efficient manner $(4,5)$. Furthermore, technological advances should be used as an adjunct to clinical effectiveness and have proven to provide better continuity of care than verbal/written handover $(5,6,7)$. However, larger studies would be required to assess whether the changes enforced by the surgical proforma affect patient morbidity or mortality rates.

\section{References}

(1) The Royal College of Surgeons of England (2011). The Higher Risk General Surgical Patient: towards improved care for a forgotten group, RCSE, London. Available at: http://www.rcseng.ac. uk/publications/docs/higher-risk-surgical-patient

(2) The Royal College of Surgeons of England (2007). Safe Handover: guidance from the Working Time Directive Working Party, RCSE, London. Available at: http://www.rcseng.ac.uk/publications/docs

(3)Freemantle N, Richardson M, Wood J, et al. (2012) Weekend hospitalization and additional risk of death: An analysis of inpatient data. J R Soc Med. Feb;105(2):74-84. Epub 2012 Feb 2.

(4) General Medical Council. Leadership and management for all doctors (2012). Available at: http://www.gmc-uk.org/Leadership_an d_management_for_all_doctors_FINAL.pdf_47234529.pdf.

(5) Matic J, Davidson P and Salamonson Y. (2009) Review:bringing patient safety to the forefront through computerisation during clinical handover. Journal of Clinical Nursing. 20, 184-189.

(6) Thompson JE, Collett LW, Langbart MJ, Purcell NJ et al. Using the ISBAR handover tool in junior medical officer handover: a study in Australian tertiary hospital. Postgrad Med J 2011 87:340-344

(7) Raptis DA, Fernandes C, Chua W \& Boulos PB (2009).

Electronic software significantly improves quality of handover in a London teaching hospital. Health Informatics J. 15(3), 191-198 